\title{
Improved Parameter Estimation in Rayleigh Model
}

\author{
Smail Mahdi ${ }^{1}$
}

\begin{abstract}
In this paper we describe and present results on the parameter point estimation for the scale and threshold parameters of the Rayleigh distribution. Five estimating methods have been investigated, namely, the maximum likelihood, the method of moment, the probability weighted moments method, the least square method and the least absolute deviation method. Modified maximum likelihood estimators for the parameters are also proposed. Simulation studies have shown that the modified likelihood estimator outperforms the estimators obtained with the other methods except in the case of very small samples.
\end{abstract}

\section{Introduction}

The two-parameter Rayleigh distribution is a continuous probability distribution which usually arises when a two dimensional vector has its two orthogonal components normally and independently distributed. The Euclidean norm of the vector will then have a Rayleigh distribution. The distribution may also arise in the case of random complex numbers whose real and imaginary components are normally and independently distributed. The modulus of these numbers will then be Rayleigh distributed. The Rayleigh variable $X$ with threshold parameter $\varepsilon$ and scale parameter $\delta$ is characterized by the cumulative function

$$
F(x)=1-e^{-\frac{(x-\mathcal{E})^{2}}{2 \delta^{2}}} \text { for } \varepsilon \leq x<\infty \text { and } \delta>0 .
$$

This distribution plays an important in real life applications since it relates to a large number of distributions such as generalized extreme value, Weibull, chisquare and rice distributions. In this paper we investigate the estimation of the scale and threshold parameters using a modified maximum likelihood method (ML), the moment method (MM), the probability weighted moment method

\footnotetext{
${ }^{1}$ Department of Computer Science, Mathematics and Physics, University of the West Indies, Cave Hill Campus, Barbados.
} 
(PWM), the ordinary least square method (OLS) and the least absolute deviation (LAD) method. The PWM method is a relatively recent technique which is well used by hydrologists in frequency analysis. The method is strongly advocated in Hosking et al. (1985) and according to Davison and Smith (1990) it constitutes the most serious competitor to the ML method, especially, in the case of small samples. The performance of this technique has been recently investigated in Mahdi and Ashkar (2004) and Ashkar and Mahdi (2003) in Weibull and Loglogistic models, respectively. We organise this paper as follows. In Section 1, we have introduced the problem and in Section 2, we derive the parameter estimators using the considered methods and also give results on the asymptotic variances. Simulations results are discussed and illustrated in Section 3.

\section{Estimation methods}

We derive and present below estimators for the parameters $\varepsilon$ and $\delta$ by using the five considered methods. We start with the probability weighted moments method.

\subsection{Probability weighted moments}

The probability weighted moment of order $(i, j, k)$ is obtained from the inverse cumulative function $x(F)=\varepsilon+\delta \sqrt{-2 \ln (1-F)}$

as

$$
\mathrm{M}_{i, j, k}=\mathrm{E}\left(X^{i}[F(x)]^{j}[1-F(x)]^{k}\right)=\int_{0}^{1}(x(F))^{i} F^{j}(1-F)^{k} d F .
$$

We use the usual orders $i=1$ and $\mathrm{j}=0$ since this leads to a class of linear Lmoments, see, Hosking $(1986 ; 1990)$, with asymptotic normality. We denote $\alpha_{r}$ the corresponding probability weighted moment $M_{1,0, r}$. After integration and simplification, we obtain

$$
\alpha_{r}=\left[\varepsilon+\delta \sqrt{\frac{\pi}{2(r+1)}}\right] /(r+1) .
$$

Substituting two distinct orders $r$ and $s$ into equation (2.2) gives the probability weighted moment estimate for $\delta$ as

$$
\hat{\delta}=\frac{(r+1) \hat{\alpha}_{r}-(s+1) \hat{\alpha}_{s}}{\sqrt{\frac{\pi}{2(r+1)}}-\sqrt{\frac{\pi}{2(s+1)}}}
$$


where $\hat{\alpha}_{r}=n^{-1} \sum_{i=1}^{n} \frac{C_{r}^{n-i} x_{i}}{C_{r}^{n-1}}$ for $r=0,1, \ldots, n-1$, with the convention $C_{j}^{k}=0$ if $k<j$, is the unbiased estimators for $\alpha_{r}$, see Hosking (1989). Thus, the estimator for $\varepsilon$ is given by

$$
\hat{\varepsilon}=(r+1) \hat{\alpha}_{r}-\hat{\delta} \sqrt{\frac{\pi}{2(r+1)}}
$$

\subsubsection{Asymptotic variances of $\hat{\varepsilon}$ and $\hat{\delta}$}

The asymptotic variances of $\hat{\varepsilon}$ and $\hat{\delta}$ are approximated by using the asymptotic variances of the PWM estimates $\hat{\alpha}_{r}$. We will use result (5.3), provided in Hosking (1986), stating that the vector whose $\mathrm{r}^{\text {th }}$ component is $\sqrt{n}\left(\hat{\alpha}_{r}-\alpha_{r}\right)$, for $r=0,1, \ldots, m-1$, has a Gaussian limiting distribution with mean vector $\mathbf{0}$ and covariance matrix $\mathrm{A}=\left(\mathrm{A}_{r s}\right)_{r, s=0}^{m-1}$ where $A_{r s}=I_{r s}+I_{s r}$ and

$$
I_{r s}=\iint_{x<y}(1-F(x))^{r}(1-F(y))^{s} F(x)(1-F(x)) d x d y .
$$

Using the approximation 26.2.10 in Abramowitz and Stegun (1970: 932), we get after integration and simplification,

$$
I_{r s}=2 \delta^{2} \sqrt{\frac{\pi}{s+1}}\left(\frac{1}{2}\left[\sqrt{\frac{\pi}{4 r}}-\sqrt{\frac{\pi}{4(r+1)}}\right]-\frac{1}{2^{r+1}}+\sum_{n=1}^{\infty} \frac{[n(2 n+1)(n-1)]^{-1}(-1)^{n}}{2^{\frac{2 n+3}{2}}(s+1)^{\frac{2 n+1}{2}}}\left[\frac{1}{(r+1)^{n+1}}-\frac{1}{r^{n+1}}\right]\right)
$$

The first order approximations for the variances and covariance of $\hat{\varepsilon}$ and $\hat{\delta}$ are obtained from the equation

$$
\operatorname{Cov}(\hat{a}, \hat{s})=n^{-1} G A G^{T}
$$

where the terms of the 2 by 2 matrix G, derived from the probability weighted moment equations of the form (2.3) and (2.4), are given by

$$
G_{11}=\frac{\partial \varepsilon}{\partial \alpha_{r}}=r+1
$$




$$
\begin{gathered}
G_{12}=\frac{\partial \varepsilon}{\partial \alpha_{s}}=0, \\
G_{21}=\frac{\partial \delta}{\partial \alpha_{r}}=\frac{r+1}{\sqrt{\frac{\pi}{2(r+1)}-\sqrt{\frac{\pi}{2(s+1)}}}},
\end{gathered}
$$

and

$$
G_{22}=\frac{\partial \delta}{\partial \alpha_{s}}=-\frac{s+1}{\sqrt{\frac{\pi}{2(r+1)}-\sqrt{\frac{\pi}{2(s+1)}}}}
$$

\subsection{Maximum likelihood}

Setting to zero the first derivative of the log-likelihood function with respect to $\delta$ gives the ML estimate for $\delta$ for a given value $\varepsilon$ as

$$
\hat{\delta}=\sqrt{\frac{\sum_{i=1}^{n}\left(x_{i}-\varepsilon\right)^{2}}{2 n}} .
$$

The maximum likelihood estimate for the parameter $\varepsilon$ which is on boundary of the distribution support is given by $\hat{\varepsilon}=\min \left(x_{1}, x_{2}, \ldots, x_{n}\right)$. Note that this estimator is biased since $\hat{\varepsilon}$ is distributed as a Rayleigh variable with threshold parameter $\varepsilon$ and scale parameter $\delta / \sqrt{n}$. To prove that, let us denote $G$ the cumulative function of $\hat{\varepsilon}$ which is based on the distribution random sample $X_{1}, \ldots, X_{n}$. The function $G$, evaluated at $\hat{\varepsilon}=y$, is given by

$$
\begin{aligned}
G(y) & =P\left(\min \left(X_{1}, \ldots, X_{n}\right) \leq y\right)=1-P\left(\min \left(X_{1}, \ldots, X_{n}\right)>y\right) \\
& =1-\left\{\left(P\left(X_{1} \succ y\right) \times \ldots \times P\left(X_{n} \succ y\right)\right\}=1-[1-F(y)]^{n} .\right.
\end{aligned}
$$

Substituting now $F(y)$ from equation (1.1), we get

$$
G(y)=1-e^{-\frac{(y-\varepsilon)^{2}}{2(\delta / \sqrt{n})^{2}}}
$$


which has the same form as $F(y)$. Therefore the Rayleigh variable $\hat{\varepsilon}$ has the mean $\varepsilon+\frac{\delta}{\sqrt{n}} \sqrt{\pi / 2}$. We propose then to use the modified maximum likelihood estimators $\tilde{\mathcal{E}}$ and $\tilde{\delta}$ that are solutions of the systems of equations

$$
\left\{\begin{array}{l}
\tilde{\mathcal{\varepsilon}}=\hat{\varepsilon}-\frac{\tilde{\delta}}{\sqrt{n}} \sqrt{\pi / 2} \\
\tilde{\delta}=\sqrt{\frac{\sum_{i=1}^{n}\left(x_{i}-\tilde{\mathcal{\varepsilon}}\right)^{2}}{2 n}}
\end{array}\right.
$$

and which are based on the unbiased estimator $\widetilde{\mathcal{E}}$. Squaring equation (2.13) and expressing $\tilde{\mathcal{E}}$ as function of $\hat{\varepsilon}$ from equation (2.12) yield the second order equation of $\tilde{\delta}$,

$$
\left(2-\frac{\pi}{2 n}\right) \tilde{\delta}^{2}+2 \sqrt{\frac{\pi}{2 n}}(\hat{\varepsilon}-\bar{x}) \tilde{\delta}+2 \bar{x} \hat{\varepsilon}-\overline{x^{2}}-\hat{\varepsilon}^{2}=0 .
$$

The discriminant of the above equation is positive and is given by

$$
\Delta=2(\hat{\varepsilon}-\bar{x})^{2}+\left(\overline{x^{2}}-\bar{x}^{2}\right)\left(2-\frac{\pi}{2 n}\right) \geq 0
$$

Therefore, equation (2.14) has two distinct solutions. Furthermore this equation admits a unique positive solution since the roots product is $-\frac{(\hat{\varepsilon}-\bar{x})^{2}+\left(\overline{x^{2}}-\bar{x}^{2}\right)}{2-\frac{\pi}{2 n}} \prec 0$. Thus the modified estimators for $\delta$ and $\varepsilon$ are respectively given by

$$
\widetilde{\delta}=\frac{\left(\bar{x}-x_{(1)}\right) \sqrt{\frac{\pi}{2 n}}+\sqrt{2\left(x_{(1)}-\bar{x}\right)^{2}+\left(\overline{x^{2}}-\bar{x}^{2}\right)\left(2-\frac{\pi}{2 n}\right)}}{2-\frac{\pi}{2 n}} \succ 0
$$

and 


$$
\widetilde{\varepsilon}=x_{(1)}-\frac{\tilde{\delta}}{\sqrt{n}} \sqrt{\pi / 2}
$$

where $\bar{x}$ and $\overline{x^{2}}$ are the first and second sample moments, respectively and $x_{(1)}$ is the first order statistic based on the random sample $x_{1}, \ldots, x_{n}$.

\subsubsection{Variance of $\hat{\delta}$ and $\tilde{\varepsilon}$.}

The asymptotic variance of $\tilde{\delta}$ is approximately given by

$$
\operatorname{Var}(\hat{\delta})=\left[E\left[-\frac{\partial^{2} \ln (f)}{(\partial \delta)^{2}}\right]\right]^{-1}=\frac{\delta^{2}}{n\left(4+\frac{3 \varepsilon^{2}}{\delta^{2}}+\frac{6 \varepsilon \sqrt{\frac{\pi}{2}}}{\delta}\right)}
$$

obtained from the sample Fisher information on $\delta$. On the other hand, we have that $\operatorname{Var}(\widetilde{\varepsilon})=\operatorname{Var}(\hat{\varepsilon})=(2-\pi / 2) \delta^{2} / n$ by using the distribution of $\hat{\varepsilon}$. Thus, $\tilde{\varepsilon}$ is a consistent estimator of $\varepsilon$.

\subsection{Method of moments}

The moment about the origin of order $r$ is given by,

$$
\mu_{\mathrm{r}}=E\left(X^{r}\right)=\int_{\varepsilon}^{\infty} x^{r} \frac{(x-\varepsilon)}{\delta^{2}} e^{-\frac{(x-\varepsilon)^{2}}{2 \delta^{2}}} d x
$$

After integration we get

$$
\begin{aligned}
\mu_{r}= & \frac{\sqrt{2}}{2 \delta} \sum_{k=0}^{r+1} C_{k}^{r+1} \varepsilon^{r+1-k}(\sqrt{2} \delta)^{k} \Gamma[(k+1) / 2] \\
& -\frac{\sqrt{2} \varepsilon}{2 \delta} \sum_{k=0}^{r} C_{k}^{r} \varepsilon^{r-k}(\sqrt{2} \delta)^{k} \Gamma[(k+1) / 2] .
\end{aligned}
$$

This can be simplified as

$$
\mu_{r}=\sum_{k=1}^{r+1} C_{k-1}^{r} \varepsilon^{r+1-k}(\sqrt{2} \delta)^{k-1} \Gamma[(k+1) / 2] .
$$


The first and second order non central moments can be evaluated from either equation (2.20) or (2.21). Using for instance equation (2.21), we get

$$
\mu_{1}=2 \varepsilon+\sqrt{2} \Gamma(3 / 2) \delta-\varepsilon=\varepsilon+\delta \sqrt{\frac{\pi}{2}}
$$

and

$$
\mu_{2}=\varepsilon^{2}+2 \sqrt{2} \Gamma(3 / 2) \varepsilon \delta+2 \delta^{2}=\varepsilon^{2}+\sqrt{2 \pi} \varepsilon \delta+2 \delta^{2}
$$

This yields the following moment method estimators for $\delta$ and $\varepsilon$,

$$
\hat{\delta}=\sqrt{\frac{n \sum_{i=1}^{n} x_{i}^{2}-\left(\sum_{i=1}^{n} x_{i}\right)^{2}}{2 n^{2}\left\{1-\frac{\pi}{4}\right\}}}=\frac{\mathrm{s}}{\sqrt{2-\frac{\pi}{2}}}
$$

and

$$
\hat{\varepsilon}=\bar{x}-\hat{\delta} \sqrt{\frac{\pi}{2}}
$$

where $s=\sqrt{\overline{x^{2}}-\bar{x}^{2}}$ is an estimator of the population standard deviation.

\subsubsection{Asymptotic variances and covariance of $\hat{\varepsilon}$ and $\hat{\delta}$}

The asymptotic variances and covariance of $\hat{\varepsilon}$ and $\hat{\delta}$ are estimated from the variances and covariance of the sample general moments $\hat{\mu}_{r}$ and $\hat{\mu}_{s}$, see for instance, Mahdi and Ashkar (2004), as follows:

$$
\left[\begin{array}{c}
\operatorname{Var}(\hat{\varepsilon}) \\
\operatorname{Var}(\hat{\boldsymbol{\delta}}) \\
\operatorname{Cov}(\hat{\varepsilon}, \hat{\delta})
\end{array}\right]=\left[\begin{array}{ccc}
M_{11}^{2} & M_{12}^{2} & 2 M_{11} M_{12} \\
M_{21}^{2} & M_{22}^{2} & 2 M_{21} M_{22} \\
M_{11} M_{21} & M_{12} M_{22} & M_{11} M_{22}+M_{21} M_{12}
\end{array}\right]^{-1}\left[\begin{array}{c}
\operatorname{Var}\left(\hat{\mu}_{r}\right) \\
\operatorname{Var}\left(\hat{\mu}_{l}\right) \\
\operatorname{Cov}\left(\hat{\mu}_{r}, \hat{\mu}_{l}\right)
\end{array}\right]
$$

where

$$
\begin{aligned}
& \operatorname{Var}\left(\hat{\mu}_{r}\right)=\frac{\mu_{2 r}-\left(\mu_{r}\right)^{2}}{n} ; \operatorname{Var}\left(\hat{\mu}_{l}\right)=\frac{\mu_{2 l}-\left(\mu_{l}\right)^{2}}{n} ; \operatorname{Cov}\left(\hat{\mu}_{r}, \hat{\mu}_{l}\right)=\frac{\mu_{r+l}-\mu_{r} \mu_{l}}{n} ; \\
& M_{11}=\frac{\partial \mu_{r}}{\partial \varepsilon} ; M_{12}=\frac{\partial \mu_{r}}{\partial \delta} ; M_{21}=\frac{\partial \mu_{l}}{\partial \varepsilon} \text { and } M_{22}=\frac{\partial \mu_{l}}{\partial \delta} .
\end{aligned}
$$

In the considered case $r=1$ and $l=2$, we have 


$$
\begin{gathered}
\operatorname{Var}\left(\hat{\mu}_{1}\right)=\frac{(4-\pi)}{2 n} \delta^{2}, \\
\operatorname{Var}\left(\hat{\mu}_{2}\right)=\frac{2(4-\pi) \delta^{2} \varepsilon^{2}+2 \sqrt{2 \pi} \delta^{3} \varepsilon+4 \delta^{4}}{n}, \\
\operatorname{Cov}\left(\hat{\mu}_{1}, \hat{\mu}_{2}\right)=\frac{\delta^{2}\left[(4-\pi) \varepsilon+\delta \sqrt{\frac{\pi}{2}}\right]}{n}, \\
M_{11}=1, \\
M_{12}=\sqrt{\frac{\pi}{2}}, \\
M_{21}=2 \varepsilon+\delta \sqrt{2 \pi}
\end{gathered}
$$

and finally:

$$
M_{22}=4 \delta+\varepsilon \sqrt{2 \pi}
$$

\subsection{Regression methods}

The parameters $\delta$ and $\varepsilon$ can also be estimated through the linear regression technique from the relation $x=\varepsilon+\delta \sqrt{-2 \ln (1-F(x)}$. Ordinary least square estimates as well as least absolute deviation estimates for $\delta$ and $\mathcal{E}$ are obtained from the sample points $\left\{x_{i}, y_{i}\right\}$ where $x_{i}$ is the $\mathrm{i}^{\text {th }}$ sample value corresponding to the empirical quantile $\hat{F}\left(x_{(i)}\right)$ and $y_{i}=\sqrt{-2 \ln \left(1-\hat{F}\left(x_{i}\right)\right.}$. Ordinary least square estimates for $\delta$ and $\varepsilon$ are obtained from the usual intercept and slope linear regression estimates, see, for instance Rice (1995). The least absolute deviation or median regression estimates of $\delta$ and $\varepsilon$ are obtained as solution to the minimization problem: $\quad \operatorname{Min} \sum_{i=1}^{n}\left|y_{i}-a-b x_{i}\right|$ with respect to $a$ and $b$. The solution is obtained by applying the simplex method to the linear programming problem:

$\operatorname{Min} \sum_{i=1}^{n}\left(r_{i}^{+}+r_{i}^{-}\right)$under the constraints $y_{i}-a-b x_{i}-r_{i}^{+}+r_{i}^{-}=0$ where $\quad r_{i}^{+} \quad$ and $r_{i}^{-}$are, respectively, the positive and negative residuals associated with the observation $x_{i}, i=1, \ldots, n$. 


\subsubsection{Variances of OLS estimators}

The computation of the variances of the least absolute deviation estimators is extremely tedious. However, we can find the variances of the ordinary least square estimators under the assumptions of the standard statistical models, see, for instance Rice (1995). Let us denote $\hat{\delta}_{\text {Ols }}$ and $\hat{\varepsilon}_{\text {Ols }}$ the OLS estimators of $\delta$ and $\varepsilon$, respectively. The variances of these estimators are, respectively, given by

$$
\operatorname{Var}\left(\hat{\delta}_{\text {Ols }}\right)=-\frac{n \sigma^{2}}{\left.2 n \sum_{i=1}^{n} \ln \left(1-\hat{F}\left(x_{i}\right)\right)+\left[\sum_{i=1}^{n} \sqrt{-2 \ln \left(1-\hat{F}\left(x_{i}\right)\right)}\right)^{2}\right]^{2}}
$$

and

$$
\operatorname{Var}\left(\hat{\varepsilon}_{\text {Ols }}\right)=\frac{2 \sigma^{2} \sum_{i=1}^{n} \ln \left(1-\hat{F}\left(x_{i}\right)\right)}{\left.2 n \sum_{i=1}^{n} \ln \left(1-\hat{F}\left(x_{i}\right)\right)+\left[\sum_{i=1}^{n} \sqrt{-2 \ln \left(1-\hat{F}\left(x_{i}\right)\right)}\right)^{2}\right]^{2}}
$$

where

$$
\sigma^{2}=\operatorname{Var}(X)=\frac{4-\pi}{2} \delta^{2}
$$

is obtained from equations (2.22) and (2.23).

\section{Discussion}

We have assessed the performance of the considered estimation methods through simulation studies. Different values of the parameters have been considered as well as different sample sizes. Orders $(1,2),(1,3)$ and $(2,3)$ are used in the PWM method. The sample points were generated using the inverse cumulative function technique. The probability weighted moments are estimated with the plotting method outlined in Hosking (1986), that is, $M_{1, u, v}$ is estimated by

$$
\hat{M}_{1, u, v}=n^{(-1)} \sum_{i} x_{(i)} p_{i}{ }^{u}\left(1-p_{i}\right)^{v}
$$

where 


$$
p_{i}=\frac{i+\gamma}{n+\delta^{\prime}} \text { for } \delta^{\prime}>\gamma>-1 .
$$

We used the values $\gamma=-0.35$ and $\delta^{\prime}=0$ which are recommended in Hosking (1986) for the study of the generalized extreme value distribution since the Rayleigh is well related to it. Several values for $\delta, \varepsilon$ and $n$ were considered, namely, $\delta=2,4,6,8,10 ; n=10,20,30,40,50,60,70,80,90,100$, and $\varepsilon=1,3,5,7,9$ and 11. Small sample sizes from 1 to 9 were also considered and obtained corresponding results are displayed in Table 3. The root mean square errors (RMSE) for the estimates were then computed and used as performance index. Note that expressions for the asymptotic variances are also obtained whenever it is possible. These variances may be used, for instance, to compute approximate confidence bounds for the underlying parameters. First we have found that the

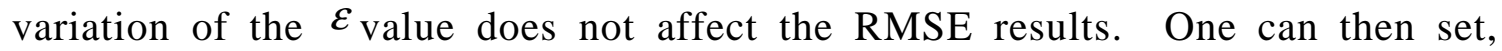
without loss of generality, $\varepsilon=1$. However, the root mean square errors obtained with all methods increase as the value $\delta$ increases, as illustrated in Table 1 below. On the other hand, the study has shown that the PWM orders 1 and 2 provide better RMSE results.

Table 1: RMSE of $\delta$ and $\varepsilon$ estimates obtained with the different methods combined by averaging the sample sizes $\mathrm{n}=10,20,30,40,50$, $60,70,80,90$ and 100 for various values of $\delta . \varepsilon=1$ and PWM orders 1 and 2 are used.

\begin{tabular}{|c|c|c|c|c|c|}
\hline$\delta$ & 2 & 4 & 6 & 8 & 10 \\
\hline$\hat{\delta}_{P W M}$ & $\begin{array}{l}.2 \\
3\end{array}$ & $\begin{array}{l}.4 \\
5\end{array}$ & .68 & .90 & $\begin{array}{l}1.1 \\
3\end{array}$ \\
\hline$\hat{\varepsilon}_{P W M}$ & $\begin{array}{l}.2 \\
5 \\
\end{array}$ & $\begin{array}{l}.5 \\
0 \\
\end{array}$ & .75 & .99 & $\begin{array}{l}1.2 \\
5 \\
\end{array}$ \\
\hline$\hat{\delta}_{M L}$ & $\begin{array}{l}.2 \\
0\end{array}$ & $\begin{array}{l}.4 \\
0\end{array}$ & .61 & .82 & $\begin{array}{l}1.0 \\
3\end{array}$ \\
\hline$\hat{\varepsilon}_{M L}$ & $\begin{array}{l}.2 \\
3\end{array}$ & $\begin{array}{l}.4 \\
7\end{array}$ & .71 & .95 & $\begin{array}{l}1.1 \\
9\end{array}$ \\
\hline$\hat{\boldsymbol{\delta}}_{m m}$ & $\begin{array}{l}.2 \\
3 \\
\end{array}$ & $\begin{array}{l}.4 \\
8 \\
\end{array}$ & .72 & .95 & $\begin{array}{l}1.2 \\
0 \\
\end{array}$ \\
\hline$\hat{\varepsilon}_{M M}$ & $\begin{array}{l}4 \\
4 \\
\end{array}$ & $\begin{array}{l}8 \\
8 \\
\end{array}$ & $\begin{array}{l}1.3 \\
1 \\
\end{array}$ & $\begin{array}{l}1.7 \\
5 \\
\end{array}$ & $\begin{array}{l}2.2 \\
0 \\
\end{array}$ \\
\hline$\hat{\delta}_{\text {OLS }}$ & $\begin{array}{l}.2 \\
3\end{array}$ & $\begin{array}{l}.4 \\
6\end{array}$ & .69 & .92 & $\begin{array}{l}1.1 \\
6\end{array}$ \\
\hline$\hat{\varepsilon}_{\text {OLS }}$ & $\begin{array}{l}.2 \\
8\end{array}$ & $\begin{array}{l}.5 \\
6 \\
\end{array}$ & .84 & $\begin{array}{l}1.1 \\
2\end{array}$ & $\begin{array}{l}1.4 \\
1 \\
\end{array}$ \\
\hline$\hat{\delta}_{L A D}$ & $\begin{array}{l}.2 \\
3\end{array}$ & $\begin{array}{l}.4 \\
6\end{array}$ & .70 & .93 & $\begin{array}{l}1.1 \\
7\end{array}$ \\
\hline$\hat{\varepsilon}_{L A D}$ & $\begin{array}{l}.2 \\
8 \\
\end{array}$ & $\begin{array}{l}.5 \\
6 \\
\end{array}$ & .85 & $\begin{array}{l}1.1 \\
4 \\
\end{array}$ & $\begin{array}{l}1.4 \\
2\end{array}$ \\
\hline
\end{tabular}

Our investigation has also shown that the method of moments performs poorly in comparison to the other methods. Table 2, displayed below, gives the root mean 
square errors as functions of the sample size $n$. It shows that the root mean square values are monotonically decreasing with the sample size n. Overall, all methods have performed reasonably well except the method of moments. However, the modified maximum likelihood method provides better estimates for $\delta$, with any sample size, and for both $\delta$ and $\varepsilon$ parameters when the sample sizes are not small, say $n \geq 10$, as illustrated in Tables 2 and 3 . Note that in the case of small samples, the PWM method outperforms the maximum likelihood method for the estimation of $\varepsilon$ and performs almost as good as the maximum likelihood method for the estimation of $\delta$, see, Table 3 results. Consequently, we recommend using the modified maximum likelihood method for the parameter estimation of the Rayleigh distribution in the case of non small samples. However, we notice that there is a gain in using the PWM method for estimating the Rayleigh threshold parameter when the sample size is small; this confirms Davison and Smith (1990) statement.

Table 2: RMSE of $\delta$ and $\varepsilon$ estimates, obtained with the different methods, combined by averaging over the values $\delta=2,4,6,8$ and 10 for various sample sizes n. $\varepsilon=1$ and PWM orders 1 and 2 are used.

\begin{tabular}{|l|l|l|l|l|l|l|l|l|l|l|}
\hline $\mathbf{n}$ & $\mathbf{1 0}$ & $\mathbf{2 0}$ & $\mathbf{3 0}$ & $\mathbf{4 0}$ & $\mathbf{5 0}$ & $\mathbf{6 0}$ & $\mathbf{7 0}$ & $\mathbf{8 0}$ & $\mathbf{9 0}$ & $\mathbf{1 0 0}$ \\
\hline$\hat{\boldsymbol{\delta}}_{P W M}$ & 1.34 & .96 & .78 & .68 & .61 & .57 & .52 & .48 & $\begin{array}{l}.4 \\
6\end{array}$ & .43 \\
\hline$\hat{\varepsilon}_{P W M}$ & 1.45 & 1.05 & .86 & .75 & .67 & .61 & .57 & .53 & $\begin{array}{l}.5 \\
0\end{array}$ & .46 \\
\hline$\hat{\delta}_{M L}$ & 1.25 & .87 & .71 & .61 & .55 & .49 & .46 & .43 & $\begin{array}{l}.4 \\
1\end{array}$ & .39 \\
\hline$\hat{\varepsilon}_{M L}$ & 1.46 & 1.03 & .82 & .70 & .62 & .56 & .52 & .48 & $\begin{array}{l}.4 \\
5\end{array}$ & .43 \\
\hline$\hat{\delta}_{m m}$ & 1.45 & 1.01 & .82 & .71 & .64 & .58 & .54 & .50 & $\begin{array}{l}.4 \\
7\end{array}$ & .45 \\
\hline$\hat{\varepsilon}_{M M}$ & 2.96 & 1.93 & 1.52 & $\begin{array}{l}1.2 \\
8\end{array}$ & 1.13 & 1.02 & .93 & .86 & $\begin{array}{l}.8 \\
1\end{array}$ & .76 \\
\hline$\hat{\delta}_{O L S}$ & 1.37 & .98 & .80 & .70 & .63 & .57 & .53 & .50 & $\begin{array}{l}.4 \\
7\end{array}$ & .44 \\
\hline$\hat{\varepsilon}_{O L S}$ & 1.70 & 1.19 & .97 & .84 & .75 & .69 & .64 & .60 & $\begin{array}{l}.5 \\
6\end{array}$ & .53 \\
\hline$\hat{\delta}_{L A D}$ & 1.40 & .99 & .80 & .70 & .62 & .57 & .53 & .49 & $\begin{array}{l}.4 \\
7\end{array}$ & .44 \\
\hline$\hat{\varepsilon}_{L A D}$ & 1.77 & 1.21 & .98 & .84 & .75 & .68 & .63 & .59 & $\begin{array}{l}.5 \\
6\end{array}$ & .53 \\
\hline
\end{tabular}

\section{Acknowledgment}

The financial support of UWI is gratefully acknowledged. The author is grateful to the editors and referees for their valuable help and suggestions that benefited and improved this article. 
Table 3: RMSE of $\delta$ and $\varepsilon$ estimates, obtained with the different methods, combined by averaging over the values $\delta=2,4,6,8$ and 10 for small values $\mathrm{n}=5,6,7,8$ and 9 . $\varepsilon=1$ and PWM orders 1 and 2 are used.

\begin{tabular}{|l|l|l|l|l|l|}
\hline \multicolumn{1}{|c|}{$\mathrm{n}$} & 5 & 6 & 7 & 8 & 9 \\
\hline$\hat{\delta}_{P W M}$ & 1.8 & 1.7 & 1.5 & 1.4 & 1.4 \\
\hline$\hat{\varepsilon}_{P W M}$ & \multicolumn{1}{c|}{5} & 0 & 8 & 9 & 1 \\
\hline$\hat{\delta}_{M L}$ & 1.9 & 1.8 & 1.7 & 1.6 & 1.5 \\
\hline$\hat{\varepsilon}_{M L}$ & 4 & 1.6 & 1.5 & 1.4 & 1.3 \\
& 2.4 & 2.1 & 1.9 & 1.7 & 3 \\
\hline$\hat{\delta}_{m m}$ & 0 & 4 & 4 & 9 & 6 \\
\hline$\hat{\varepsilon}_{M M}$ & 0 & 1.9 & 1.7 & 1.6 & 1.5 \\
& 4.6 & 4.1 & 3.7 & 3.4 & 3.1 \\
\hline$\hat{\delta}_{O L S}$ & 7 & 3 & 3 & 2 & 7 \\
\hline$\hat{\varepsilon}_{O L S}$ & 4 & 1.9 & 1.6 & 1.5 & 1.4 \\
& 2.4 & 2.2 & 2.0 & 1.9 & 1.8 \\
\hline$\hat{\delta}_{L A D}$ & 9 & 4 & 6 & 1 & 0 \\
\hline$\hat{\varepsilon}_{L A D}$ & 2.0 & 1.8 & 1.6 & 1.5 & 1.4 \\
& 3 & 3 & 7 & 8 & 8 \\
\hline
\end{tabular}

Note: The numerical studies have been carried out with Gauss and SPSS, Release 11.

\section{References}

[1] Abramowitz, M. and Stegun, I. (1970): Handbook of Mathematical Functions. New York: Dover Publications, Inc.

[2] Ashkar, F. and Mahdi, S. (2003): Comparison of two fitting methods for the log-logistic distribution. Water Resources Research, 39, no. 8.

[3] Davison, A.C. and Smith, R.L. (1990): Models for exceedances over high threshold. J. R. Stat. Soc. B, 52 (3), 393-442.

[4] Hosking, J.R.M. (1986): The Theory of Probability Weighted Moments. New York. Research Report RC12210, IBM Thomas J. Watson Research Center.

[5] Hosking, J.R.M. (1990): L-Moments: analysis and estimation of distributions using linear combinations of order statistics. J. R. Stat. Soc. B, 52(1), 105124.

[6] Mahdi, S. and Ashkar, F. (2004): Exploring Generalized Probability Weighted Moments, Generalized Moments and Maximum Likelihood Estimating Methods in Two-Parameter Weibull Model. Journ. of Hydrology, 285, 62-77.

[7] Rice, J.A. (1995): Mathematical Statistics and Data Analysis, $2^{\text {nd }}$ Ed. Duxbury Press. 